\title{
The Hidden Value of Assessing Right Ventricular Performance with Exercise in Hypertensive Patients with Left Ventricular Diastolic Dysfunction
}

\author{
Mohamed Sanhoury, Samir Rafla, Tarek El Badawy, Radwa Momtaz Khalil \\ Cardiology Department, Faculty of Medicine, Alexandria University, Alexandria, Egypt \\ Email: drsanhory@yahoo.com,smrafla@yahoo.com, tarekelbadawy@gmail.com,radwa.momtaz@yahoo.com
}

\begin{abstract}
How to cite this paper: Sanhoury, M., Rafla, S., El Badawy, T., \& Khalil, R. M. (2021) The Hidden Value of Assessing Right Ventricular Performance with Exercise in Hypertensive Patients with Left Ventricular Diastolic Dysfunction. World Journal of Cardiovascular Diseases, 11, 261-276.

https://doi.org/10.4236/wjcd.2021.116026
\end{abstract}

Received: April 23, 2021

Accepted: June 12, 2021

Published: June 15, 2021

Copyright $\odot 2021$ by author(s) and Scientific Research Publishing Inc. This work is licensed under the Creative Commons Attribution International License (CC BY 4.0).

http://creativecommons.org/licenses/by/4.0/

(c) (i) Open Access

\begin{abstract}
Background: Right ventricular (RV) dysfunction could develop during exercise in patients with both hypertension and left ventricular diastolic dysfunction and may contribute to the patient symptoms. The objective is to assess $\mathrm{RV}$ function, both at rest and during exercise in patients with hypertension and left ventricular diastolic dysfunction. Methods: We included 30 patients with hypertension and resting LV diastolic dysfunction. The systolic function of the right ventricle was assessed by TAPSE (Tricuspid Annular Plane Systolic Excursion) and S, while E/A ratio, annular lateral E', E/E' and E'/A' were used to measure diastolic function. The global function of the right ventricle was assessed by measuring the right indexed myocardial performance. The dimensions and pulmonary pressures were also measured. Results: The following parameters of RV systolic function were increased significantly with exercise: TAPSE $(P=0.0054), S^{\prime}(P=0.0045)$. Moreover, the following diastolic parameters of the RV increased significantly with exercise: $\mathrm{E} / \mathrm{E}^{\prime}(\mathrm{P}=$ $0.05), A^{\prime}(P=0.04)$. The global RV function showed also a significant increase $(\mathrm{P}=0.0011)$. The three RV dimensions as well as the pulmonary artery pressures also increased during exercise $(\mathrm{P}=0.000004,0.001$, and 0.00000064 respectively). In addition, the presence of resting LV grade II DD predicted significantly higher pulmonary pressures during exercise $(P=0.006)$. The advanced resting grade of LVDD predicted significantly the presence of advanced grade of RVDD with exercise $(P=0.037)$. Conclusions: Some patients who have both hypertension and LV diastolic dysfunction showed structural and functional changes of the right ventricle at rest. However, all patients had $\mathrm{RV}$ functional changes during exercise.
\end{abstract}




\section{Keywords}

Right Ventricular Dysfunction, Left Ventricular Dysfunction, Exercise, Echocardiography, Tricuspid Annular Plane Systolic Excursion (TAPSE)

\section{Introduction}

The impact of hypertension on the LV is very well known. However, little evidence is known about changes in the right ventricular systolic and diastolic function in patients with both hypertension and left ventricular diastolic dysfunction [1] [2] [3] [4] [5]. These changes can be attributed to: 1) Increased activity of both sympathetic and renin-angiotensin-aldosterone (RAAS) system [6] [7] [8] . 2) Hypertrophy of the septum and RV wall thickening [9] [10] [11].

Exercise is known to be a very useful technique to detect and magnify many cardiac changes.

It has been used for:

- Clarification of the presence of ischemic heart disease.

- Evaluation of both systemic and pulmonary artery pressures which may be normal at rest.

- Monitoring of both systolic and diastolic function during exercise.

- Evaluation of physical fitness as an initial step for medical evaluation of sports practice and prescription of cardiac rehabilitation programs [12] [13] [14] [15].

RV function increases with exercise according to Frank-starling law, due to increased preload and mild dilation of RV dimensions [16].

Aim: To assess the performance of the right ventricle, at rest and with exercise, in patients with both hypertension and left ventricular diastolic dysfunction.

Patients and Methods: Study population.

Inclusion criteria: The present study included 30 patients presented to hypertension clinic, with normal sinus rhythm, and whose transthoracic echo revealed evidence of left ventricular diastolic dysfunction, a normal systolic function of both left and right ventricles.

Exclusion criteria: We excluded patients with myocardial ischemia (based on symptoms, resting electrocardiogram (ECG) or/and normal coronary angiography if available, more than a mild disease of the heart valves, diabetes mellitus (DM), evidence of pulmonary hypertension on resting TTE, evident right ventricular pathology on resting TTE, severe tricuspid regurgitation (TR), or musculoskeletal problem precluding exercise.

Methods: All the study participants have signed informed consent. And the study was approved by the ethical committee. All the patients and control were subjected to full history taking, including age, gender, cardiovascular disease risk factors. 
Laboratory investigation: Lipid profile, urea, and creatinine were measured in all patients.

Resting transthoracic echocardiography: The following echocardiographic parameters were measured.

Left ventricle: Four parameters were used to diagnose diastolic dysfunction in patients with preserved ejection fraction (LVEF) [13]: Average E/E', septal E', lateral E' velocities by TDI at the mitral annulus, peak tricuspid regurgitation velocity, and left atrial volume index (LAVI). M-Mode was used to measure LVEF [17].

Devereux formula was used to measure the Left ventricular mass (LVM). LVH was graded according to 2015 recommendations on the use of echocardiography in adults with hypertension [18]. Relative wall thickness (RWT) was calculated using the following formula ( $\left.2^{\star} \mathrm{PWT} / \mathrm{LVEDD}\right), \mathrm{PWT}$ is the posterior wall thickness and LVEDD is the left ventricular end-diastolic dimension [18].

Right ventricle:

- Systolic function was assessed by TAPSE (transannular plane systolic excur-

sion) using M-Mode, and TDI velocities as TASV: tricuspid annular systolic velocity [19];

- Diastolic function was assessed using E/A ratio using conventional pulsed wave;

Doppler, E' by TDI, E/E' ratio.

Tricuspid flow velocities are assessed by pulsed-wave Doppler in the apical four-chamber view. The following variables were determined: early diastolic peak flow velocity (E wave); late diastolic flow velocity (A wave).

TDI was used to measure myocardial velocities of the RV in the apical four-chamber view, with a sample volume placed at the tricuspid annulus lateral segment during systole (S') and early diastole (E') and tricuspid (E/E') ratio was measured [19] [20] [21].

The RV diastolic function was assessed, and the grading was done according to 2010 guidelines for the echocardiographic assessment of the right heart in adults [19].

The RV MPI (RV myocardial performance index or the Tei index) was obtained by tissue Doppler method which allows measurement of MPI as well as $S^{\prime}$, $\mathrm{E}^{\prime}$, and A', all from a single image [20].

$(\mathrm{RV}$ MPI $=($ IVCT + IVRT $) /$ RVET $)$ (where IVCT is the isovolumetric contraction time, IVRT is the isovolumetric relaxation time; RVET is the right ventricular ejection time in $\mathrm{msec}$ ). RV dimensions were measured in the apical four-chamber view. 3 measurements were taken: basal RV diameter at the level of tricuspid annulus, RV mid-cavity, and longitudinal diameter (from apex to annulus).

PAT (pulmonary acceleration time) was also recorded using pulsed-wave Doppler at the RV outlet (RVOT) in a short-axis view.

Symptom limited treadmill stress test (modified Bruce protocol): 
The following information was collected: resting heart rate, maximum symptom-limited heart rate, resting systolic blood pressure and diastolic blood pressure, maximum metabolic equivalent achieved at the peak of exercise. Echocardiography after treadmill stress test: An echocardiography study was performed immediately after the exercise using the same parameters previously measured.

\section{Statistical Analysis}

Data were analyzed using the Statistical Package for Social Sciences (SPSS ver20 Chicago, IL, USA). K-S test was done to check for normality of quantitative variables. Normally distributed data were expressed using mean, standard deviation (SD), minimum (min), and maximum ( $\max )$. Paired t-test to analyze the significance of the effect of exercise on the echocardiographic parameters (numerical variables), independent $t$-test to compare echocardiographic parameters (numerical variables) among groups. Qualitative/Categorical data were described using number and percent. To compare $2 \times 2$ categorical variables, the Chi-square test was used, but the Fisher Exact test of significance (FE, non-parametric test) was used when $>$ twenty percent of the cells had expected cell count less than 5. Mont-Carlo test was used instead of the Fisher test when tables were more than $2 \times 2(2 \times 3)$. Pearson correlation $(r)$ was utilized to the strength of association of 2 continuous variables [15].

Research protocol approved from our institutional review board (IRB) and human subject informed consent.

\section{Results}

\section{Baseline Characteristics}

The patients' mean age was $50 \pm 7.1$ years old ( $35-63) .30 \%$ of the patients were smokers. $36.6 \%$ were receiving angiotensin-converting enzyme inhibitors (ACEI), $20 \%$ of them were receiving B-Blockers, $6 \%$ of them were receiving calcium channel blockers (CCB), $13.3 \%$ of them were receiving diuretic therapy and $24.7 \%$ of them were receiving combination therapy.

Clinical characteristics: they are shown in Table 1.

Electrocardiogram: All the patients had normal sinus rhythm on their resting ECG with no ischemic changes or arrhythmias. Laboratory Investigations: they

Table 1. Clinical characteristics of the study patients.

\begin{tabular}{cccccccccc}
\hline $\begin{array}{c}\text { Clinical } \\
\text { Characteristics }\end{array}$ & Resting HR & $\begin{array}{c}\text { Exercise } \\
\text { HR }\end{array}$ & $\begin{array}{c}\text { Resting } \\
\text { SBP }\end{array}$ & $\begin{array}{c}\text { Resting } \\
\text { DBP }\end{array}$ & $\begin{array}{c}\text { Exercise } \\
\text { SBP }\end{array}$ & $\begin{array}{c}\text { Exercise } \\
\text { DBP }\end{array}$ & $\begin{array}{c}\text { Mets } \\
\text { Achieved }\end{array}$ & $\begin{array}{c}\text { exercise } \\
\text { duration } \\
(\mathrm{min})\end{array}$ \\
\hline $\begin{array}{c}\text { Mean } \\
\text { Standard } \\
\text { deviation }\end{array}$ & 88.0 & 126.33 & 128.3 & 82.67 & 155.5 & 93.33 & 5.15 & 6.5 \\
$\begin{array}{c}\text { Maximum } \\
\text { Minimum }\end{array}$ & 11.2 & 15.42 & 9.7 & 7.27 & 14.9 & 11.643 & 1.94 & 0.157 \\
\hline
\end{tabular}


are shown in Table 2.

Echocardiography:

Table 3 and Table 4 described the echocardiographic parameters of the patients at rest and exercise, respectively.

The following echocardiographic parameters showed significant changes during exercise (using paired t-test analysis):

Left ventricle: Systolic function:

- LVEF significantly increased with exercise $(\mathrm{P}$ value $=0.00000011)$, Diastolic function: the following parameters were significantly increased with exercise;

- Doppler-measured trans-mitral E and A waves ( $P$ value $=0.04499$ and 0.00000045 , respectively).

- Septal and lateral mitral E’ waves (P value $0.000006 \& 0.000549$, respectively)

- The average left ventricular E/E' (LV E/E') significantly increased with exercise $(\mathrm{P}$ value $=0.004)$.

- Left atrial volume index LAVI significantly increased with exercise ( $\mathrm{P}$ value $=$ 0.0004).

Right ventricle: Systolic function:

TAPSE and S' significantly increased with exercise ( $\mathrm{P}$ value $=0.00018$ and 0.0065 , respectively).

Diastolic function: Both RV E/E' ratio and A' significantly increased with exercise ( $\mathrm{P}$ value $=0.049$ and 0.048 , respectively) .

Global function: RIMP was significantly increased with exercise ( $\mathrm{P}$ value $=$ $0.0012)$, while PAT was significantly decreased with exercise $(\mathrm{P}$ value $=0.00000067)$. All three measured right ventricular dimensions were significantly increased with exercise.

The following pairs of echocardiographic parameters showed statistically significant moderate correlations: Resting $\mathrm{E} / \mathrm{A}$ ratio of the $\mathrm{LV}$ with resting $\mathrm{E} / \mathrm{A}$ ratio of the RV $(r=0.353, P$ value $=0.0495)$, exercise LV E/E' ratio to exercise RIMP $(r=+0.4203, \mathrm{P}$ value $=0.021)$ and exercise LV E/E' ratio to exercise PAT $(\mathrm{r}=-0.4011, \mathrm{P}$ value $=0.028000578)$.

In our study, 14 (46\%) patients had LVH and 16 (53\%) patients had no LVH. The subgroup with LVH included 8 males and 6 females, with LVM (Left ventricular mass): $175 \pm 8.8 \mathrm{~g}$ in females, $256 \pm 12.9 \mathrm{~g}$ in males, RWT was $0.46 \pm$ 0.02 .

Table 2. Laboratory investigations of the study patients.

\begin{tabular}{ccccccc}
\hline Lab. results & $\mathrm{Hb}$ & $\begin{array}{c}\text { Platelets } \\
(\times 1000)\end{array}$ & WBC & Creatinine & Na & K \\
\hline Mean & 12.9 & 293 & 6083 & 1.04 & 141 & 4.04 \\
Max. & 14.5 & 430 & 10,000 & 1.4 & 148 & 4.7 \\
Min. & 11.2 & 162 & 3950 & 0.7 & 136 & 3.4 \\
SD & 0.89 & 74 & 1692 & 0.205 & 3.6 & 0.388 \\
Median & 13 & 290 & 5674 & 1 & 141 & 3.9 \\
\hline
\end{tabular}


Table 3. Echocardiographic parameters of the study patients at rest.

\begin{tabular}{|c|c|c|c|c|c|}
\hline Parameter & Mean & Min & $\operatorname{Max}$ & $\begin{array}{l}\text { Standard } \\
\text { Deviation }\end{array}$ & Abnormal if \\
\hline \multicolumn{6}{|l|}{ Left ventricle } \\
\hline LV EF & $61 \%$ & $50 \%$ & $75 \%$ & $7 \%$ & $(<50 \%)$ \\
\hline EDD & 51.16 & 42 & 58 & 4.44 & $\begin{array}{l}>60 \mathrm{~mm}(\mathrm{M}),>53 \\
\mathrm{~mm}(\mathrm{~F})\end{array}$ \\
\hline ESD & 33.06 & 25 & 40 & 5.71 & $>40 \mathrm{~mm}$ \\
\hline Septal wall thickness (SWT) & 10.63 & 7 & 13 & 1.683 & $>10 \mathrm{~mm}$ \\
\hline Posterior wall thickness (PWT) & 9.7 & 6 & 12 & 1.636 & $>10 \mathrm{~mm}$ \\
\hline $\mathrm{E}(\mathrm{m} / \mathrm{sec})$ & 0.585 & 0.26 & 0.9 & 0.189 & \\
\hline $\mathrm{A}(\mathrm{m} / \mathrm{sec})$ & 0.75 & 0.52 & 1.2 & 0.167 & \\
\hline $\mathrm{E} / \mathrm{A}$ & 0.78 & 0.46 & 1.34 & 0.203 & $\begin{array}{l}<0.8 \rightarrow \text { G I-DD } \\
0.8-2 \rightarrow \text { G II-DD } \\
>2 \rightarrow \text { G III-DD }\end{array}$ \\
\hline E/E' (average) & 7.58 & 3.1 & 13.2 & 3.059 & $\begin{array}{l}<10 \rightarrow \text { G I-DD } \\
10-14 \rightarrow \text { G II-DD } \\
>14 \rightarrow \text { G III-DD }\end{array}$ \\
\hline Septal E' $(\mathrm{cm} / \mathrm{sec})$ & 5.69 & 4.5 & 6.8 & 0.611 & $<7 \mathrm{~cm} / \mathrm{sec}$ \\
\hline Lateral E' (cm/sec) & 8.92 & 6 & 14 & 1.901 & $<10 \mathrm{~cm} / \mathrm{sec}$ \\
\hline LAVI & 38.86 & 34.5 & 48.7 & 3.977 & $>34 \mathrm{~mm}$ \\
\hline \multicolumn{6}{|l|}{ Right ventricle } \\
\hline TAPSE $(\mathrm{cm})$ & 2.243 & 1.7 & 2.9 & 0.37 & $<1.7 \mathrm{~cm}$ \\
\hline $\mathrm{S}^{\prime}(\mathrm{TDI})(\mathrm{cm} / \mathrm{sec})$ & 13.8 & 9.6 & 19 & 2.33 & $<9.5 \mathrm{~cm} / \mathrm{sec}$ \\
\hline $\mathrm{E} / \mathrm{A}$ & 0.986 & 0.4 & 1.88 & 0.279 & $<0.8$ or $>2.0$ \\
\hline $\mathrm{E}^{\prime}$ (TDI) & 13.29 & 5.3 & 84 & 13.39 & $<7.8$ \\
\hline $\mathrm{E} / \mathrm{E}^{\prime}$ & 4.39 & 2.3 & 8.3 & 1.485 & $>6$ \\
\hline$A^{\prime}$ & 13.993 & 8.5 & 24 & 3.457 & $>15$ \\
\hline $\mathrm{E}^{\prime} / \mathrm{A}^{\prime}$ & 0.8 & 0.46 & 1.23 & 0.223 & $<0.52$ \\
\hline Global RV function RIMP (TDI) & 0.4 & 0.215 & 0.57 & 0.09 & $>0.54$ \\
\hline annular RV dimension & 34.57 & 23 & 40 & 4.255 & $>41$ \\
\hline Mid cavity RV dimension & 27.8 & 20 & 35 & 4.59 & $>35$ \\
\hline longitudinal RV dimension & 67.9 & 47 & 80 & 10.6 & $>83$ \\
\hline PAT & 175.7 & 116 & 270 & 50.23 & $<100$ \\
\hline
\end{tabular}

The subgroup without LVH included 7 males and 9 females, with LVM: $129 \pm$ $13 \mathrm{~g}$ in females, $193.7 \pm 19.6 \mathrm{~g}$ in males, RWT was $0.33 \pm 0.03$.

Resting-state: 1) Left ventricle:

All patients had a preserved ejection fraction (EF) with a mean value of $61 \% \pm$ $7 \%$.

Regarding the diastolic function, 18 patients $(60 \%)$ had left ventricular grade I 
Table 4. Echocardiographic parameters of the study patients with exercise.

\begin{tabular}{|c|c|c|c|c|c|}
\hline Parameter & Mean & Min & $\operatorname{Max}$ & $\begin{array}{l}\text { Standard } \\
\text { Deviation }\end{array}$ & Abnormal if \\
\hline \multicolumn{6}{|l|}{ Left ventricle } \\
\hline LV EF & $66 \%$ & $50 \%$ & $84 \%$ & $9 \%$ & $(<50 \%)$ \\
\hline EDD & 50.93 & 39 & 58 & 4.9 & $\begin{array}{l}>60 \mathrm{~mm} \text { (male) } \\
>53 \mathrm{~mm} \text { (female) }\end{array}$ \\
\hline ESD & 31.66 & 23 & 39 & 4.61 & $>40 \mathrm{~mm}$ \\
\hline SWT & 10.63 & 7 & 14 & 1.853 & $>10 \mathrm{~mm}$ \\
\hline PWT & 9.63 & 6 & 13 & 1.816 & $>10 \mathrm{~mm}$ \\
\hline $\mathrm{E}$ & 0.683 & 0.1 & 1.2 & 0.257 & \\
\hline A & 0.89 & 0.09 & 1.6 & 0.333 & \\
\hline $\mathrm{E} / \mathrm{A}$ & 0.758 & 0.5 & 1.33 & 0.206 & $\begin{array}{l}<0.8 \rightarrow \text { GI-DD } \\
0.8-2 \rightarrow \text { GII-DD } \\
>2 \rightarrow \text { GIII-DD }\end{array}$ \\
\hline E/E' (average) & 8.613 & 4.8 & 13.2 & 2.456 & $\begin{array}{l}<10 \rightarrow \text { GI-DD } \\
10-14 \rightarrow \text { GII-DD } \\
>14 \rightarrow \text { GIII-DD }\end{array}$ \\
\hline Septal E' & 6.83 & 4.8 & 11 & 1.205 & $<7$ \\
\hline Lateral E' & 10.1 & 7 & 14.5 & 2.214 & $<10$ \\
\hline LAVI & 41.09 & 34 & 52.7 & 4.539 & $>34$ \\
\hline \multicolumn{6}{|l|}{ Right ventricle } \\
\hline TAPSE $(\mathrm{cm})$ & 2.496 & 1.9 & 3.1 & 0.322 & $<1.7 \mathrm{~cm}$ \\
\hline $\mathrm{S}^{\prime}(\mathrm{TDI})(\mathrm{cm} / \mathrm{sec})$ & 15.72 & 10.5 & 26 & 3.948 & $<9.5 \mathrm{~cm} / \mathrm{sec}$ \\
\hline $\mathrm{E} / \mathrm{A}$ & 0.913 & 0.4 & 1.25 & 0.226 & $<0.8$ or $>2.0$ \\
\hline $\mathrm{E}^{\prime}(\mathrm{TDI})$ & 10.91 & 6 & 25 & 4.44 & $<7.8$ \\
\hline $\mathrm{E} / \mathrm{E}^{\prime}$ & 5.28 & 1.79 & 9.6 & 2.335 & $>6$ \\
\hline$A^{\prime}$ & 15.607 & 10 & 27 & 4.243 & $>15$ \\
\hline$E^{\prime} / A^{\prime}$ & 0.694 & 0.42 & 1.4 & 0.222 & $<0.52$ \\
\hline $\begin{array}{l}\text { Global RV function } \\
\text { RIMP (TDI) }\end{array}$ & 0.655 & 0.219 & 1.7 & 0.368 & $>0.54$ \\
\hline $\begin{array}{l}\text { annular RV } \\
\text { dimension }\end{array}$ & 37.12 & 26 & 46 & 4.9 & $>41 \mathrm{~mm}$ \\
\hline $\begin{array}{l}\text { Mid cavity RV } \\
\text { dimension }\end{array}$ & 30.9 & 22 & 42 & 5.267 & $>35 \mathrm{~mm}$ \\
\hline $\begin{array}{l}\text { longitudinal RV } \\
\text { dimension }\end{array}$ & 71.84 & 50 & 86 & 10.11 & $>83 \mathrm{~mm}$ \\
\hline PAT & 138 & 80 & 240 & 46.66 & $<100$ \\
\hline
\end{tabular}

$\mathrm{PAT}=$ Pulmonary acceleration time.

diastolic dysfunction and 12 (40\%) patients had left ventricular grade II diastolic dysfunction.

LVH and LV Diastolic function grade at rest:

- Among the patients without LVH (16 patients), 13 Patients had LV GI-DD, 
and 3 patients had LV GII-DD;

- Among the patients with LVH, 5 Patients had LV GI-DD, and 9 patients had LV GII DD.

The difference of the effect of LVH on the grade of the LV diastolic function was statistically significant ( $\mathrm{P}$ value 0.013 , using fisher exact test), showing higher incidence of GIIDD among patients with LVH.

Furthermore, among patients with LVH (14 patients), 8 patients had mild LVH, and 6 patients had moderate LVH. Among patients with mild LVH, 8 patients had LV GIDD, and no one had LV GII-DD. Among patients with moderate LVH, there were 2 patients with LV GI-DD, and 4 patients with LVGII-DD.

When comparing the subgroups with mild and moderate LVH, there was a statistically significant correlation between the severity of LVH and the grade of LVDD ( $P$ value $=0.014985$ ), using FE test, showing a higher incidence of an advanced grade of diastolic dysfunction among the subgroup with moderate LVH.

2) Right ventricle: In all patients, TAPSE and RV S' were preserved. The mean TAPSE was $2.19 \pm 1.3 \mathrm{~mm}$, the mean RV S' was $13.5 \pm 7.5 \mathrm{~cm} / \mathrm{sec}$.

The diastolic function is described as follows: 7 patients $(23.3 \%)$ had normal $\mathrm{RV}$ diastolic function, all of them were found to be in the subgroup without LVH. 17 patients (56.6\%) had grade I RV GI-DD (Among them 8 patients (26.67\%) were in the subgroup with LVH, 7 patients $(23.33 \%)$ with mild LVH, and one patient $(3.33 \%)$ with moderate $\mathrm{LVH}$ ) and 9 patients $(32.67 \%)$ had no LVH). 6 (20\%) patients had RV GIIDD, all of them were in the subgroup with LVH. 5 patients (16.67\%) with moderate LVH and one of them (3.33\%) had mild LVH.

LVH and RV Diastolic function at rest: By applying the FE test, there was a statistically significant correlation between the presence of LVH and the presence of RVDD at rest $(\mathrm{P}$ value $=0.005)$. Among patients with LVH and RVDD (14 patients, there was a statistical significance between the severity of LVH and the grade of RVDD at rest $(P$ value $=0.016)$.

RIMP: All cases with RVDD (23 patients) showed also RIMP alteration (increased more than 0.54 by TDI at rest).

3) Correlation between RV and LV function at rest:

Systolic function: There was a weak positive correlation between the RV and LV systolic function $(r=0.096)$.

Diastolic function:

1) In patients with LV GI-DD (18 patients (60\%)): 6 patients $(20 \%)$ had normal RV Diastolic function, 9 patients (30\%) had RV GI-DD, and 3 patients (10\%) had RV GII-DD. In patients with LV GII-DD (12 patients (40\%)), only one patient (3.33\%) had normal RV Function, 8 patients (26.66\%) had RV GI-DD, and 3 patients (10\%) had RV GII-DD.

2) At rest, there was no statistically significant difference between the two grades of LVDD in the incidence of RVDD (P value $=0.109$ ).

3) Furthermore, the patients who had RVDD (23 patients) had almost equal 
numbers of LV GI-DD and LV GII-DD cases (12 cases vs 11 cases). Therefore, among patients with RVDD (23 patients), there was no statistically significant difference between the two grades of LVDD in the incidence of a corresponding/certain grade of RVDD ( $\mathrm{P}$ value $=0.36$.

With Exercise:

1) Left ventricle: LV systolic function:

In all patients, EF increased significantly during exercise (P 0.00000011).

LV diastolic function: Among patients without LVH (16 patients), 2 Patients had LV GI-DD, 13 patients had LV GII-DD and 1 patient had LV GIII-DD. Among patients with LVH (14 patients), 8 patients had mild LVH, and 6 patients had moderate LVH. Among patients with mild LVH, 2 patients had LV GI-DD, 6 patients had LV GII-DD. Among patients with moderate LVH, 5 patients had LV GII-DD and one patient had LV GIII-DD.

When comparing the subgroup with mild LVH and the subgroup with moderate $\mathrm{LVH}$, there was no statistical difference between the 2 groups in the incidence of a certaingrade of left diastolic dysfunction during exercise ( $\mathrm{P}$ value $=$ $0.35)$.

Moreover, when comparing the LVH group to the non-LVH group, there was no difference between the 2 groups in the incidence of a certain grade of LVDD during exercise. $(\mathrm{P}$ value $=0.4$ )

Regarding the 18 patients who had LV GI-DD at rest: 4 patients (13.33\%) of the sample remained as LV GI-DD and 14 patients (46.66\%) developed LV GII-DD at exercise.

Regarding the 12 patients who had LV GII-DD at rest: 10 patients (33.33\%) remained as LV GIIDD with exercise, and 2 patients (6.66\%) developed G III DD.

2) Right ventricle:

RV systolic function: In all patients, TAPSE and RV S' both increased significantly during exercise, ( $\mathrm{P} 0.005$ and 0.0045 respectively).

RV diastolic function: Exercise RVDD was altered in all patients. Ten patients (33.33\%) had exercise RV GI-DD. Among them, 6 patients (20\%) were in the subgroup without $\mathrm{LVH}, 2$ patients (6.67\%) were in the subgroup with mild LVH, and 2 patients (6.67\%) were in the subgroup with moderate LVH. 18 patients (60\%) had RV GII-DD. Among them, 8 of them (26.67\%) had no LVH, 6 patients (20\%) were in the subgroup with mild LVH, and 4 patients (13.33\%) were in the subgroup with moderate LVH. 2 patients (6.67\%) had RV G III-DD; both were in the subgroup without LVH.

There was no statistical significance between the presence of LVH and the incidence of RVDD at exercise $(\mathrm{P}$ value $=0.22)$. Among patients with LVH and associated exercise RVDD (14 patients), there no statistically significant association between the severity of $\mathrm{LVH}$ and the grade of RV diastolic dysfunction grade at exercise $(\mathrm{P}$ value $=0.428)$.

Regarding the 7 patients (23.33\%) who had resting normal RV diastolic function, 3 patients remained with normal RV diastolic function, 4 (13.34\%) patients 
developed RV GI-DD and 3 (10\%) patients developed RV GII-DD.

Regarding the 17 patients (56.67\%) who had resting RVGI-DD, 6 patients (20\%) remained to have RV GI-DD and 11 patients (36.66\%) developed RV GII-DD.

- Regarding the 6 patients (20\%) who had resting RV GII-DD, 4 (13.34\%) of them remained to have RV GII-DD (E/E'> 6, E/A = $0.8-2)$ and $2(6.67 \%)$ patients developed RV GIII-DD. Therefore, the distribution of the status of $\mathrm{RV}$ diastolic function of the cases with exercise was as follows: no patient had normal RV diastolic function, 10patients had RV GI-DD, 18 patients had RV GII-DD and 2 patients had RV GIII-DD.

Global RV function:

As all the cases had exercise RVDD (30 patients), they also showed a significant increase of exercise RIMP (TDI), compared with resting RIMP ( $\mathrm{P}$ value $=$ $0.001)$.

3) Correlation between RV and LV during exercise:

Systolic function: There was a positive moderate correlation between RV and LV systolic function during exercise $(r=0.33)$, which was not detected at rest.

Diastolic function: All 30 patients had abnormal RV diastolic function during exercise.

1) The 4 patients (13.33\%): who had LV GI-DD during exercise, developed also RV GIDD during exercise.

2) The 24 patients (80\%) who had exercise LV GII-DD were distributed as follows during exercise: 4 patients developed RV GI-DD, 18 patients developed RV GII-DD and 2 patients developed RV GIII-DD.

3) The 2 patients (6.66\%) who had LV GIII-DD during exercise, developed RV GI-DD during exercise.

In patients with LV GI-DD and LV GII-DD during exercise, the grade of LVDD predicted significantly the incidence of the corresponding grade of exercise RVDD ( $P$ value $=0.0046)$.

- In addition, the presence of resting LV GII-DD predicted significantly higher pulmonary pressures during exercise $(\mathrm{P}=0.006)$. Furthermore, the advanced resting grade of LVDD predicted significantly the presence of advanced grade of RVDD with exercise $(\mathrm{P}$ value $=0.037)$.

\section{Discussion}

We studied the right ventricular performance during exertion in a group of hypertensive individuals with resting left ventricular diastolic dysfunction. We observed that among patients with LVH, the difference in the effect of LVH on the grade of the LVDD was statistically significant (P value 0.013 ). Furthermore, among patients with LVH, when comparing the subgroup with mild LVH and the subgroup with moderate $\mathrm{LVH}$, there was a statistically significant correlation between the grade of the severity of LVH and the grade of LVDD.

Ventricular diastolic dysfunction can be present even in the absence of overt 
LVH in hypertensive patients. This can be caused by concentric remodeling especially in those with long = standing hypertension. This remodeling can be associated with normal or small LV cavity size decreased LV compliance and increase stiffness.

In our study, the resting systolic function of the right ventricle was preserved in all patients. This can be explained by the absence of resting overt pulmonary hypertension in these cases.

TM Gorter et al. did a meta-analysis on RV dysfunction in heart failure with preserved ejection fraction (HFpEF). They observed that RV dysfunction is mainly associated with high pulmonary artery pressures, AF, and reduced left ventricular systolic function. These criteria were excluded in our study and this may explain the preserved RV systolic function in our study [16].

According to the previously mentioned meta-analysis, RV systolic dysfunction is more prevalent in males as well as those with more severe degrees of left ventricular diastolic dysfunction, ischemic artery disease, and higher BMI [16].

In our study, the presence of LVH predicted significantly the incidence of RVDD at rest. Among patients with LVH and associated RVDD, the severity of LVH predicted significantly the grade of RVDD grade at rest.

In general, the presence of RVDD can be explained by the diastolic ventricular interdependence (shared pericardium) and the same fibrotic process involving both ventricles [16]. Furthermore, in our study, the resting E/A ratio of both ventricles had a significant moderate correlation at rest. Moreover, the LVH is normally associated with neurohormonal changes, sympathetic overflows, and activation of the RAAS system which may affect both ventricles.

Cicala et al. used tissue Doppler to study right ventricular diastolic dysfunction in 30 normotensive and 30 hypertensive patients. They found that hypertension leads to RV longitudinal diastolic dysfunction (in the form of prolongation of active relaxation as a result of ventricular interaction from left ventricular pressure overload conditions) [17].

Tumuklu MM1, et al used RV standard Doppler indices, myocardial Doppler imaging, and strain/strain rate imaging indices to study the impact of both hypertension and its related LVH on the functions of the right ventricle. They found that the peak systolic strain of the RV free wall was lower than control in addition to significant alteration of the RV diastolic measurements [18].

Morris et al studied right ventricular dysfunction (both systolic and diastolic) in patients with heart failure and preserved LVEF. They found that RV systolic and diastolic dysfunction are common and possibly related to the same fibrotic processes that affect the LV and to a lesser extent with RV pressure overload [19].

In our study, all cases with resting RVDD showed also resting RIMP alteration (by TDI).

\subsection{Ventricular Interdependence}

Systolic ventricular interdependence: 
In the present study, a weak positive correlation between the RV and LV systolic function was observed. Around $20 \%-40 \%$ of RV systolic performance can be attributed to LV contraction due to sharing myocardial fibers and septum between both ventricles. This is well known as systolic ventricular interdependence.

Diastolic ventricular interdependence:

In our study, at rest, there was no statistically significant difference between the two grades of LVDD in the incidence of RVDD. Furthermore, among patients who had RVDD, no statistically significant difference between the two grades of LVDD in the incidence of a corresponding/certain grade of RVDD was observed.

Also, patients who had RVDD had an almost equal number of LV GI DD and LV GII DD (12 cases vs 11 cases). However, the incidence of RV GI-DD was higher than the incidence of RV GII-DD. This may be due to increased compliance of the RV walls compared to LV walls.

Left ventricular function with exercise:

LV systolic function with exercise: In all patients, the preserved EF increased significantly during exercise. This can be explained by the increased sympathetic activation during exercise (inotropic effect, increased preload).

Furthermore, the worsening LV diastolic function during exercise generates higher Enddiastolic pressure during exercise, leading to excessive activation of the compensatory mechanisms. (Frank-starling law), and therefore increasing the stroke volume.

\subsection{Diastolic Function with Exercise}

In our study, when comparing the subgroup with mild LVH and the subgroup with moderate LVH, there was no statistical difference between the 2 groups in the incidence of a certain grade of left diastolic dysfunction during exercise. Furthermore, when comparing the LVH group to the non-LVH group, there was no statistical difference between the 2 groups in the incidence of a certain grade of LVDD during exercise.

In addition, we found a wide spectrum of diastolic function alterations during exercise even in patients with the same grade of resting LV DD.

The study conducted by Jong-Won Ha et al. has shown the value of LV diastolic function assessment during exercise. They included 120 patients with diastolic dysfunction and borderline E/e'. All patients underwent diastolic stress echocardiography. They observed marked changes during exercise and they recommended diastolic stress echocardiography as an important tool to further assess those patients [20].

Right ventricle function during exercise:

In our study, the exercise RV diastolic function was altered in all patients. There was no statistically significant association between the presence of LVH and the incidence of RVDD with exercise. 
Among patients with LVH and associated exercise RVDD, there was no statistical significance between the severity of LVH and the grade of RV diastolic dysfunction grade with exercise.

The worsening of the RV diastolic function can be explained by the diastolic ventricular interdependence (shared pericardium), as the LVDD worsened during exercise too [20].

RIMP (TDI):

As all cases had exercise RVDD, they also showed a significant increase of exercise RIMP (TDI), compared with resting RIMP. Furthermore, we found a significant correlation between RIMP \& LV E/E' during exercise.

Correlation between RV and LV during exercise:

Systolic function: There was a positive moderate correlation between RV and LV systolic function during exercise. This correlation was found to be weak at rest.

This shows the role of exercise in unmasking the moderate correlation between the two ventricles. Therefore, this unmasked systolic interdependence can be explained by the shared septum and the shared myocardial fibers between the two ventricles.

Diastolic function: All the patients had abnormal RV diastolic function during exercise. In addition, our results showed the role of exercise in unmasking the effect of LVDD on the RV diastolic function [19] [20] [21].

However, this was not applicable for G III DD of both ventricles; this could be explained by the small number of cases who developed G III DD.

Of note, in our study, the diastolic behavioral changes of both ventricles were correlated during exercise (ventricular interaction).

This was emphasized by Gorter et al. who reported that early in heart failure with preserved systolic function, RVDD could be present, at least during exercise, and in correlation with LVDD, due to possible simultaneous affection of both ventricles with the same myocardial process [21].

Effect of RV on LV: Furthermore, the relatively significant increase in RV dimensions during exercise (especially annular and mid-cavitary dimensions), as shown in Table 4, could stretch the pericardium leading to a form of pericardial constraint. The latter may in turn interfere with LV dilation \& therefore can impair the LV filling and worsen the LV diastolic dysfunction with exercise (diastolic ventricular interdependence (DVI)) [21].

Study limitation: One of the limitations of our study was the small sample size. Also, we did not use other imaging modalities such as strain echocardiography, 3D imaging.

\section{Conclusion}

Patients with hypertension and left ventricular diastolic dysfunction could have right ventricular diastolic dysfunction even in absence of left ventricular hypertrophy. However, right ventricular diastolic dysfunction grade will correlate with 
the severity of left ventricular hypertrophy as well as diastolic dysfunction. Exercise echocardiography is an important diagnostic tool to magnify the wide spectrum of left ventricular and right ventricular diastolic function alterations, as well as the correlation between them, even if not detected at rest.

\section{Conflicts of Interest}

The authors declare no conflicts of interest regarding the publication of this paper.

\section{Author Contributions}

Made the study design: MS, RK, TB. Data analysis: MS, RK, TB. Wrote the initial manuscript: MS, RK, TB. Prepared final manuscript before submission: MS, RK, TB. All authors reviewed and approved the final manuscript. SR revised and submitted the paper on behalf of the authors.

\section{References}

[1] Satpathy, C., Mishra, T.K., Satpathy, R., et al. (2006) Diagnosis and Management of Diastolic Dysfunction and Heart Failure. American Family Physician, 73, 841-846.

[2] Hosseini, S., Fazlinezhad, A., Jalalyazdi, M., et al. (2017) Diastolic Function Changes during Stress Echocardiography in Hypertensive Patients. Razavi International Journal of Medicine, 5, 6.

[3] Tarazi, R.C. (1985) The Heart in Hypertension. The New England Journal of Medicine, 312, 308-309. https://doi.org/10.1056/NEJM198501313120510

[4] Hegde, S.M. and Solomon, S.D. (2015) Influence of Physical Activity on Hypertension and Cardiac Structure and Function. Current Hypertension Reports, 17, Article No. 77. https://doi.org/10.1007/s11906-015-0588-3

[5] Cicala, S., Galderisi, P., Caso, P., et al. (2002) Right Ventricular Diastolic Dysfunction in Arterial Systemic Hypertension: Analysis by Pulsed Tissue Doppler. European Journal of Echocardiography, 3, 135-142. https://doi.org/10.1053/euje.2001.0124

[6] Rezk, A., Nouh, S.H. and Basiouny, T. (2013) Impact of Systemic Hypertension on Right Ventricular Function (Analysis by Tissue Doppler). AAMJ, 10, 70-89.

[7] Spring, A., Kosmala, W. and Jołda-Mydłowska, B. (1997) Right Ventricular Diastolic Dysfunction and Its Relation to Left Ventricular Performance in Patients with Hypertension. Polskie Archiwum Medycyny Wewnetrznej, 97, 323-332.

[8] Cotrim, C., João, I., Fazendas, P., et al. (2013) Clinical Applications of Exercise Stress Echocardiography in the Treadmill with Upright Evaluation during and after Exercise. Cardiovascular Ultrasound, 11, Article No. 26. https://doi.org/10.1186/1476-7120-11-26

[9] Nagueh, S.F., Smiseth, O.A., Appleton, C.P., et al. (2016) Recommendations for the Evaluation of Left Ventricular Diastolic Function by Echocardiography: An update from the American Society of Echocardiography and the European Association of Cardiovascular Imaging. Journal of the American Society of Echocardiography, 29, 277-314. https://doi.org/10.1016/j.echo.2016.01.011

[10] Kossaify, A. (2015) Echocardiographic Assessment of the Right Ventricle, from the Conventional Approach to Speckle Tracking and Three-Dimensional Imaging, and 
Insights into the "Right Way" to Explore the Forgotten Chamber. Clinical Medicine Insights: Cardiology, 9, 65-75. https://doi.org/10.4137/CMC.S27462

[11] Lang, M.R., Badano, L.P., Mor-Avi, V., et al. (2015) Recommendations for Cardiac Chamber Quantification by Echocardiography in Adults: An Update from the American Society of Echocardiography and the European Association of Cardiovascular Imaging. Journal of the American Society of Echocardiography, 28, 1-39. https://doi.org/10.1016/j.echo.2014.10.003

[12] Marwick, T.H., Gillebert, T.C., Aurigemma, G., et al. (2015) Recommendations on the Use of Echocardiography in Adult Hypertension: A Report from the European Association of Cardiovascular Imaging (EACVI) and the American Society of Echocardiography (ASE). Journal of the American Society of Echocardiography, 28, 727-754. https://doi.org/10.1016/j.echo.2015.05.002

[13] Rudski, L.G., Lai, W.W., Afilalo, J., et al. (2010) Handschumacher, Chandrasekaran K: Guidelines for the Echocardiographic Assessment of the Right Heart in Adults. Journal of the American Society of Echocardiography, 23, 685-713. https://doi.org/10.1016/j.echo.2010.05.010

[14] Lakoumentas, J.A., Panou, F.K., Kotseroglou, V.K., et al. (2005) The Tei Index of Myocardial Performance: Applications in Cardiology. Hellenic Journal of Cardiology, 46, 52-58.

[15] Kirkpatrick, L.A. and Feeney, B.C. (2013) A Simple Guide to IBM SPSS Statistics for Version 20.0. Student Edition, Belmont, Calif: Wadsworth, Cengage Learning.

[16] Gorter, T.M., Hoendermis, E.S., van Veldhuisen, D.J., et al. (2016) Right Ventricular Dysfunction in Heart Failure with Preserved Ejection Fraction: A Systematic Review and Meta-Analysis. European Journal of Heart Failure, 18, 1472-1487. https://doi.org/10.1002/ejhf.630

[17] Ernst, R., Schwarz, E.R. and Dashti, R (2010) The Clinical Quandary of Left and Right Ventricular Diastolic Dysfunction and Diastolic Heart Failure. Cardiovascular Journal of Africa, 21, 212-220.

[18] Tumuklu, M.M., Erkorkmaz, U. and Ocal, A. (2007) The Impact of Hypertension and Hypertension-Related Left Ventricle Hypertrophy on Right Ventricle Function. Echocardiography, 24, 374-384. https://doi.org/10.1111/j.1540-8175.2007.00419.x

[19] Morris, D.A., Gailani, M., Perez, A.V., et al. (2011) Right Ventricular Myocardial Systolic and Diastolic Dysfunction in Heart Failure with Normal Left Ventricular Ejection Fraction. Journal of the American Society of Echocardiography, 24, 886-897. https://doi.org/10.1016/j.echo.2011.04.005

[20] Ha, J.-W. (2018) Diastolic Stress Echocardiography to Quantify the Response of Diastolic Functional Indices to Dynamic Exercise in Abnormal Relaxation: Unmasking Diastolic Abnormalities Is Getting Ready for Prime Time. Korean Circulation Journal, 48, 755-759. https://doi.org/10.4070/kcj.2018.0164

[21] Gorter, T.M., van Veldhuisen, D.J., Bauersachs, J., et al. (2018) Right Heart Dysfunction and Failure in Heart Failure with Preserved Ejection Fraction: Mechanisms and Management. Position Statement on Behalf of the Heart Failure Association of the European Society of Cardiology. European Journal of Heart Failure, 20, 16-37. 


\section{Abbreviations}

TDI = Tissue Doppler Imaging; renin-angiotensin-aldosterone (RAAS); MPI (RV myocardial performance index or the Tei index); DD = Diastolic dysfunction; $\mathrm{PAT}=$ pulmonary acceleration time; RIMP $=\mathrm{RV}$ myocardial performance. 